\title{
GEOMETRIC QUANTIZATION OF A PARTICLE IN A PERPENDICULAR MAGNETIC FIELD
}

\section{GUILLERMO CAPOBIANCO AND WALTER REARTES}

\author{
Communicated by Vasil V. Tsanov
}

Abstract. In this paper we develop the quantization of a particle in the plane under the influence of a perpendicular magnetic field using the geometric quantization with half-forms in Hilbert space of holomorphic functions. An original coordinate transformation is applied to convert the problem into a system of harmonic oscillators. Then, it is solved highlighting the relationship between different representations. We emphasize the isomorphism between the holomorphic representation and the Schrödinger representation.

MSC: 53D50, 81S10

Keywords: Geometric quantization, Landau levels, Segal-Bargmann transform

\section{Introduction}

Geometric quantization is a well established theory (see for example $[5,11,15,16$, $22,25,27,31]$ ), and it is useful in the choice of coordinates and other structures such as operators, Hilbert spaces and isomorphisms between different representations.

The system studied here is a particle in a plane under the influence of a perpendicular magnetic field. This system provides the basis for analyzing other phenomena such as the quantum Hall effect [20] or the study of anyons [30]. The same system was studied in [4] with a completely different approach. Also, a different approach to the holomorphic quantization of a particle in a magnetic field is presented by Hall and Kirwin [14] or Dunne [6].

The following section briefly explains the theory of geometric quantization. We state the main ideas that lead to the quantization with half-forms, without extensive developments and demonstrations which can be found in the reference section.Then a model consisting of a charged particle moving in a plane under the influence of a perpendicular magnetic field is developed. Special emphasis is placed on the original choice of coordinates, the complex structure and description of the Hilbert space. In the literature we can find interesting examples of complex problems solved through a specific coordinate transformations [7]. 
Finally, we explicitly write the isomorphism between the holomorphic representation and the Schrödinger representation, also known as the Segal-Bargmann transform [12].

\section{Geometric Quantization}

The classical mechanical systems can be described very elegantly in the language of differentiable manifolds [1,2]. In this description, the physical states are the points of a smooth manifold $M$, known as the phase space. This manifold is equipped with a closed two-form, the symplectic form. This form gives a natural volume in the manifold called the Liouville volume. The symplectic structure results in a geometry called symplectic geometry [8], where the basic fact is not the measurement of the length, but the oriented area. Hence, it follows that symplectic forms can only exist in manifolds with even dimension.

The other important elements in the description of a classical physical system are the observables, which are differentiable functions defined on the manifold. In the set of observables there is a bilinear operation, namely the Poisson bracket, which plays a key role. This operation converts the space of smooth functions into a Poisson algebra. Also, it is worth noting the relevance of the Hamiltonian observable, which represents the energy and determines the time evolution of any observable. The time derivative of an observable is the Poisson bracket of the same observable with the Hamiltonian.

The quantization of a classical system associates it with its quantum counterpart, assigning Hermitian operators in a Hilbert space to the classical observables. This space describes the quantum states. Hermitian operators play the role of quantum observables.

Quantum observables form a non-commutative algebra, with the commutator multiplied by $-\mathrm{i} / \hbar$ as bilinear operation.

The geometric quantization $[15,22,25,27,31]$, which began with the works of Kostant and Souriau $[18,28]$, is a set of rules that associate self-adjoint operators on some Hilbert space with functions on a symplectic manifold $M$ (i.e., classical observables). This linear mapping, $f \mapsto \hat{f}$, should verify the following rules
1) $\hat{1}=\mathbb{I}$
2) $\widehat{\{f, g\}}=-(\mathrm{i} / \hbar)[\hat{f}, \hat{g}]$.

Here, 1 is the constant function, $\mathbb{I}$ is the identity operator, $\{f, g\}$ is the Poisson bracket between $f$ and $g$ and $[\hat{f}, \hat{g}]=\hat{f} \hat{g}-\hat{g} \hat{f}$ is the commutator between the 
operators $\hat{f}$ and $\hat{g}$. A map that satisfies these conditions is called a prequantization map.

Ideally, a third condition should be verified, which when $M=\mathbb{R}^{2 n}$, can be expressed as

3) canonical coordinates $q^{i}$ and $p_{i}, i=1, \ldots, n$ are represented irreducibly.

Let $A$ and $B$ be two self-adjoint operators on $H$ satisfying the canonical commutation relations $([A, B]=\mathrm{i} \hbar \mathbb{I})$. Also, suppose that $A$ and $B$ act irreducibly on $H$, meaning that the only closed subspaces of $H$ invariant under $A$ and $B$ are $\{0\}$ and $H$. Then, providing the exponentiated commutation relations hold [13], by the Stone-von Neumann theorem $[13,23], A$ and $B$ are unitarily equivalent to the operators of position and momentum, $\hat{q}$ and $\hat{p}$, i.e., there is a unitary operator $U: H \rightarrow L^{2}(\mathbb{R})$ such that $U A U^{-1}=\hat{q}$ and $U B U^{-1}=\hat{p}$.

In the Schrödinger representation of the Euclidean case, the Stone-von Neumann theorem implies that the Hilbert space consists of square integrable functions that depend only on the position, and the operators which correspond to $q$ and $p$ are the multiplication by $q$ and the derivative with respect to $q$ multiplied by $-\mathrm{i} \hbar$, respectively.

Finding an application that verifies all the conditions for all the observables is impossible. Moreover, it is not even possible in the Euclidean case restricting observables to polynomial functions, as described in the theorem of Groenewoldvan Hove [10]. Therefore, the geometric quantization program can only aspire to represent some operators. In the best case scenario, it represents a Lie subalgebra of the algebra of observables.

Geometric quantization is a two-step process. First, a Hilbert space is associated with the symplectic manifold on which the observables act as symmetric operators (prequantization). The conditions 1) and 2) are verified. The representation of the canonical coordinates is not irreducible. The reason is that the wave functions depend on the coordinates and momenta. In the second step, in order to verify the condition 3), a subspace of wave functions that depend on half of the variables is chosen. This is achieved by selecting a structure called polarization, this essentially being a choice of a maximal subspace on which the symplectic form vanishes at each point of the cotangent bundle of the phase space. 


\subsection{Prequantization}

The phase space has a natural volume, the Liouville volume, $\Omega$. If we call $\omega$ to the symplectic form, it follows that

$$
\Omega=(-1)^{\frac{n(n-1)}{2}} \frac{\omega^{n}}{n !}
$$

where $\omega^{n}$ is the exterior product of $\omega$ with itself $n$ times.

The candidate for prequantization Hilbert space is the space of square integrable complex functions with the measure given by this volume. In this space the observables act as classical Hamiltonian vector fields. A function $f$ corresponds to the unique vector field $X_{f}$ defined by $\omega\left(X_{f}, \cdot\right)=\mathrm{d} f$. The Poisson bracket between functions $f$ and $g$ is given by $\{f, g\}=\omega\left(X_{f}, X_{g}\right)$.

If we associate the operator $-\mathrm{i} \hbar X_{f}$ to the function $f$, then this map satisfies condition 2) but not condition 1), since the constants are in the kernel of the operator. One might think that modifying this operator with the addition of a term $f$, i.e., taking $-\mathrm{i} \hbar X_{f}+f$, solves the problem. Despite condition 1) being now satisfied, condition 2) is not fulfilled.

There is not a naturally associated space of complex functions. To consider complex functions we must find how to "glue" the complex plane at each point of the phase space (thought of as complex vector space of dimension one). A structure of this type is called a complex line bundle. The condition for the existence of such structure is that the integral of $\omega$ on any closed surface be an integer multiple of $2 \pi \hbar$. This condition corresponds to the quantization conditions in the early models of quantum mechanics [26]. A symplectic manifold that satisfies this condition is called quantizable.

We call $L$ to this complex line bundle. $L$ has a natural projection, assigning $m$ to every point of the complex plane on $m \in M$. The natural way to view the complex functions is through applications of $M$ in $L$ such that when composed with the projection it gives the identity on $M$. These applications are called sections of $L$.

After choosing a section $s_{0}$ of module one, i.e., $\left|s_{0}(m)\right|=1$ for all $m \in M$, any other section is written as $s(m)=\phi(m) s_{0}(m)$. In the fibers of $L$ it is defined a Hermitian scalar product given by $\left(s_{1}(m), s_{2}(m)\right)=\bar{\phi}_{1}(m) \phi_{2}(m)$. Integrating this product on $M$ we obtain a scalar product in the space of sections of $L$. The square integrable sections form a Hilbert space which is called prequantization Hilbert space.

Given a symplectic potential $\theta$, i.e., a locally defined one-form such that $\omega=\mathrm{d} \theta$, and a vector field $X$ on $M$, the following expression defines a covariant derivative of the section $s=\phi s_{0}$ in the direction of the field $X$ 


$$
\nabla_{X} s=X(\phi) s_{0}+\frac{\mathrm{i}}{\hbar} \theta(X) s_{0}
$$

where $\theta$ is called the connection one-form.

Given an observable $f$, if we define the operator

$$
\hat{f}_{P Q}=-\mathrm{i} \hbar \nabla_{X_{f}}+f
$$

where $X_{f}$ is the Hamiltonian field of $f$ and the last term is the operator of multiplication by $f$, then $\hat{f}_{P Q}$ is a symmetric operator which satisfies 1) and 2).

The expression (2) depends on the symplectic potential $\theta$ which represents the connection of the bundle in the corresponding coordinate chart. If the symplectic form is not exact, then a globally defined one-form $\theta$ cannot be defined and hence at the intersection of two coordinate charts the respective one-forms $\theta_{1}$ and $\theta_{2}$ are related by $\theta_{2}=\theta_{1}+\mathrm{d} u$, with $u$ being a locally defined function. When changing the coordinate chart, the section $s_{0}$ should be modified and this is accompanied by a change in the function $\phi$ as follows $\phi_{2}=\mathrm{e}^{\mathrm{i} u / \hbar} \phi_{1}$. The above mentioned summarizes the theory of connections on complex line bundles with the corresponding change of gauge.

At this point we note that the condition 3) is not verified. For example, in the Euclidean case with canonical coordinates $(q, p)$ the operators corresponding to $q$ and $p$ are

$$
\hat{q}_{P Q}=\mathrm{i} \hbar \frac{\partial}{\partial p}+q, \quad \hat{p}_{P Q}=-\mathrm{i} \hbar \frac{\partial}{\partial q} .
$$

This representation is not irreducible. For example, the sections that depend only on the coordinate $q$ are invariant under the action of both operators. It could be expected that restricting the space to these functions, an irreducible representation will be achieved. However, in this case there is no square integrable nonzero functions.

The next section shows how to make a map that verifies the quantization conditions by introducing a new structure (complex polarization).

\subsection{Holomorphic Quantization}

The second step in the geometric quantization is to restrict the Hilbert space of prequantization to assure that condition 3) is verified. When the manifold $M$ is $\mathbb{R}^{2 n}$, one can take the set of functions that depend only on the position. As stated above, there are no square integrable functions with the Liouville volume (unless $M$ is compact). To obtain the quantization space we can change the integration 
measure. This is done by introducing a new structure called polarization. However, the only quantizable canonical observables are those whose flow preserves the polarization.

A polarization is a subspace with half the dimension of the tangent space where the symplectic form vanishes. The polarization can be real or complex (it can also be an intermediate case). In this paper we use a complex polarization.

A complex structure $J$ is an automorphism of the tangent space of $M$ verifying $J^{2}=-I$ [17]. A structure of a complex vector space can be locally given to the tangent space by defining the product of a vector $X$ with a complex number $a+\mathrm{i} b$ as $(a+\mathrm{i} b) X=a X+b J X$. Whether this structure can be globally defined or not is related to the Nirenberg-Newlander theorem [21]. Here, we assume that the conditions of the theorem are met. In this case we have a complex manifold.

$J$ acts on the complexified tangent space, which is obtained by linear combinations of the vectors with complex coefficients. $J$ has eigenvalues i and $-i$. Moreover, the complexified tangent space is the direct sum of eigenspaces corresponding to these eigenvalues. Then, the complex vector space defined above can be identified with the eigenspace of eigenvalue $-i$ at each point $[9,17]$. Thus, there is a smooth choice of a subspace of the complexified tangent space at each point of the manifold. This is called a distribution, and this distribution is called a holomorphic polarization. $T_{\mathbb{C}} M=\mathcal{P} \oplus \overline{\mathcal{P}}, T_{\mathbb{C}} M$ is the complexified tangent space, $\mathcal{P}$ is the holomorphic polarization, and $\overline{\mathcal{P}}$ the antiholomorphic polarization.

Square integrable sections of the prequantization bundle that are covariantly constant in the directions of the antiholomorphic polarization, i.e., $\nabla_{X} s=0$ if $X \in \overline{\mathcal{P}}$, form the Hilbert space of holomorphic quantization.

\subsubsection{Half-Form Quantization}

Thus far, we have considered the wave functions as sections of the bundle of prequantization. However, if the physical interpretation is to be considered, the square modulus of the wave functions is a probability density. The latter observation motivates the introduction of an additional structure, which is the final step in the geometric quantization in most cases of physical interest. This structure is a bundle of half-forms.

First we define the canonical bundle $\mathcal{K}_{\mathcal{P}}$, comprised of the complex $n$-forms on $M$ which vanish in the fields of $\overline{\mathcal{P}}$, i.e., $\alpha(X)=0$ for all $X$ in $\overline{\mathcal{P}}$. Those that are closed, i.e., $\mathrm{d} \alpha=0$, are the holomorphic $n$-forms and are called polarized. 
If $\alpha$ and $\beta$ are elements of $\mathcal{K}_{\mathcal{P}}$ then $\bar{\alpha} \wedge \beta$ is proportional to the Liouville volume. We can define a scalar product in the fibers of $\mathcal{K}_{\mathcal{P}}$, given by

$$
\frac{1}{n !} \bar{\alpha} \wedge \beta=(-\mathrm{i})^{n}(\alpha, \beta) \Omega \text {. }
$$

In $\mathcal{K}_{\mathcal{P}}$ there are two operations, namely the Lie derivative $\mathcal{L}_{X} \alpha$, defined for fields that preserve $\overline{\mathcal{P}}$, and the partial connection $\nabla_{X} \alpha=\mathrm{d} \alpha(X)$, defined for fields in $\overline{\mathcal{P}}$.

The square root of a bundle $\mathcal{K}_{\mathcal{P}}$ is a bundle $\delta_{\mathcal{P}}$ on $M$ such that $\delta_{\mathcal{P}} \otimes \delta_{\mathcal{P}}$ is isomorphic to $\mathcal{K}_{\mathcal{P}}$ together with the corresponding isomorphism. In this work we take a cotangent bundle as phase space. Determining the existence of this bundle can be difficult; however, in this case, the bundle $\delta_{\mathcal{P}}$ exists. If $\nu_{1}$ and $\nu_{2}$ are sections of $\delta_{\mathcal{P}}$, then $\nu_{1} \otimes \nu_{2}$ is a section of $\mathcal{K}_{\mathcal{P}}$.

The Lie derivative and the partial connection may be extended to sections of the bundle $\delta_{\mathcal{P}}$ by imposing the condition $\mathcal{L}_{X}(\alpha \otimes \alpha)=2 \alpha \otimes \mathcal{L}_{X} \alpha$. From the aforementioned, it follows that there is a unique Hermitian structure on $\delta_{\mathcal{P}}$ such that

$$
(\nu, \nu)=\sqrt{(\nu \otimes \nu, \nu \otimes \nu)} .
$$

The connection in $L$ and the partial connection on $\delta_{\mathcal{P}}$ are combined to give a partial connection on $L \otimes \delta_{\mathcal{P}}$.

In the space of square integrable polarized sections of $L \otimes \delta_{\mathcal{P}}$ there is a product given by

$$
\left\langle s_{L 1} \otimes \nu_{1}, s_{L 2} \otimes \nu_{2}\right\rangle=\int_{M}\left(s_{L 1}, s_{L 2}\right)\left(\nu_{1}, \nu_{2}\right) \Omega
$$

where $s_{L i} \otimes \nu_{i}$ is a section of $L \otimes \delta_{\mathcal{P}}$, and $\left(s_{L 1}, s_{L 2}\right)$ and $\left(\nu_{1}, \nu_{2}\right)$ are Hermitian structures on $L$ and $\delta_{\mathcal{P}}$, respectively.

With all of these elements, the quantization operator with half-forms can be defined as follows: let $f$ be a classical observable whose Hamiltonian flow preserves the polarization $\mathcal{P}$ and $s=s_{L} \otimes \nu$, then the operator

$$
\hat{f}\left(s_{L} \otimes \nu\right)=\left(\hat{f}_{P Q} s_{L}\right) \otimes \nu-\mathrm{i} \hbar s_{L} \otimes \mathcal{L}_{X} \nu
$$

is a symmetric operator which verifies the quantization conditions 1) and 2). This step also verifies condition 3). Moreover, if the flow of $X_{f}$ is complete, then $\hat{f}$ is self-adjoint.

\section{Particle in a Magnetic Field}

Considering a charged particle moving in a plane under the influence of a perpendicular magnetic field, the classical phase space of the system is the cotangent 
space $M=T^{*} \mathbb{R}^{2}$. We use canonical coordinates $v=\left(q_{1}, q_{2}, p_{1}, p_{2}\right) \in \mathbb{R}^{4}$ and the canonical symplectic two-form $\omega=\mathrm{d} q_{1} \wedge \mathrm{d} p_{1}+\mathrm{d} q_{2} \wedge \mathrm{d} p_{2}$.

We consider the symmetric gauge where the components of the magnetic vector potential for the magnetic field $B$ are $A_{1}=-B q_{2} / 2$ and $A_{2}=B q_{1} / 2$, and also the classical observables $H$ and $L$ (the Hamiltonian and angular momentum). These are given by

$$
H=\frac{1}{2 m}\left(\left(p_{1}+\frac{e B}{2} q_{2}\right)^{2}+\left(p_{2}-\frac{e B}{2} q_{1}\right)^{2}\right), \quad L=q_{1} p_{2}-q_{2} p_{1}
$$

where $m$ and $e$ are the mass and the charge of the particle, respectively.

Following, with a change of canonical coordinates given by

$$
\begin{aligned}
Q_{1} & =-\frac{p_{2}}{\sqrt{e B}}+\frac{q_{1}}{2} \sqrt{e B}, & Q_{2} & =-\frac{p_{1}}{\sqrt{e B}}+\frac{q_{2}}{2} \sqrt{e B} \\
P_{1} & =\frac{p_{1}}{\sqrt{e B}}+\frac{q_{2}}{2} \sqrt{e B}, & P_{2} & =\frac{p_{2}}{\sqrt{e B}}+\frac{q_{1}}{2} \sqrt{e B}
\end{aligned}
$$

we obtain

$$
H=\frac{\omega_{c}}{2}\left(P_{1}^{2}+Q_{1}^{2}\right), \quad L=\frac{1}{2}\left(P_{2}^{2}+Q_{2}^{2}-\left(P_{1}^{2}+Q_{1}^{2}\right)\right)
$$

where $\omega_{c}=e B / m$ is the cyclotron frequency.

To obtain the quantum description of the above system we use the geometric quantization scheme. In the cotangent case, we can choose a global symplectic potential. Then, we opt for a trivial prequantization bundle given by $L=M \times \mathbb{C}$ with connection form $\Theta_{0}=P_{1} \mathrm{~d} Q_{1}+P_{2} \mathrm{~d} Q_{2}$ (the canonical one-form of the cotangent bundle).

Following, we analyze complex polarizations corresponding to complex structures in $\mathbb{R}^{4}$. We take the complex structure $J$ given by the matrix

$$
J=\left(\begin{array}{rrrr}
0 & 0 & 1 & 0 \\
0 & 0 & 0 & 1 \\
-1 & 0 & 0 & 0 \\
0 & -1 & 0 & 0
\end{array}\right)
$$

This is compatible with the symplectic structure and gives complex variables. More precisely, if we consider the projections $\Pi^{+}$and $\Pi^{-}$in the complexified phase space $\left(\mathbb{R}^{4} \otimes \mathbb{C} \equiv \mathbb{C}^{4}\right)$ given by

$$
\Pi^{ \pm}=\frac{1 \mp \mathrm{i} J}{2}
$$


and we call $V=\left(Q_{1}, Q_{2}, P_{1}, P_{2}\right)$, then we have

$$
\Pi^{+} V=z_{1} e_{1}+z_{2} e_{2}
$$

where $z_{1}=P_{1}+\mathrm{i} Q_{1}, z_{2}=P_{2}+\mathrm{i} Q_{2}, e_{1}=(-\mathrm{i}, 0,1,0) / 2=\partial / \partial z_{1}$ and $e_{2}=(0,-\mathrm{i}, 0,1) / 2=\partial / \partial z_{2}$. Applying $\Pi^{-}$, the conjugate equation is obtained, in particular $\bar{z}_{1}=P_{1}-\mathrm{i} Q_{1}$ and $\bar{z}_{2}=P_{2}-\mathrm{i} Q_{2} \cdot e_{1}$ and $e_{2}$ are a basis for the complex polarization $\mathcal{P}$, and $\bar{e}_{1}$ and $\bar{e}_{2}$ are a basis corresponding to $\overline{\mathcal{P}}$. Then, we have $\mathbb{R}^{4} \otimes \mathbb{C}=\mathcal{P} \oplus \overline{\mathcal{P}}$.

We call $z=\left(z_{1}, z_{2}\right) \in \mathbb{C}^{2}$ to the corresponding complex coordinates of the holomorphic polarization, and $|z|^{2}=z_{1} \bar{z}_{1}+z_{2} \bar{z}_{2}$. A complex manifold of this type, with a symplectic structure and a compatible metric, is called a Kähler manifold (Kobayashi and Nomizu [17]). In this type of manifolds there exists a function, the Kähler scalar, in this case given by

$$
K=\frac{1}{2}|z|^{2} .
$$

From this function, an adapted symplectic potential is obtained, and it is given by

$$
\theta_{K}=-\frac{\mathrm{i}}{2}\left(\bar{z}_{1} \mathrm{~d} z_{1}+\bar{z}_{2} \mathrm{~d} z_{2}\right)=-\mathrm{i} \partial K
$$

where $\partial$ is the operator

$$
\partial \phi=\frac{\partial \phi}{\partial z_{1}} \mathrm{~d} z_{1}+\frac{\partial \phi}{\partial z_{2}} \mathrm{~d} z_{2} .
$$

Using the above operator and its conjugate, the symplectic form is expressed as follows

$$
\omega=-\frac{\mathrm{i}}{2}\left(\mathrm{~d} \bar{z}_{1} \wedge \mathrm{d} z_{1}+\mathrm{d} \bar{z}_{2} \wedge \mathrm{d} z_{2}\right)=-\mathrm{i} \partial \bar{\partial} K
$$

Thus, we have a complex admissible positive polarization $\mathcal{P}$ with adapted oneform $\theta_{K}$. This one-form defines a global trivialization of the fiber bundle $L$ where the polarized sections are holomorphic functions of $z_{1}$ and $z_{2}$.

The observables $H$ and $L$ generate canonical fluxes which preserve the polarization and are therefore quantizable. In coordinates $(z, \bar{z})$ they are given by

$$
H=\frac{\omega_{c}}{2} z_{1} \bar{z}_{1}, \quad L=\frac{1}{2}\left(z_{2} \bar{z}_{2}-z_{1} \bar{z}_{1}\right) .
$$

We define a new symplectic potential $\theta$ given by

$$
\theta=\theta_{K}+\frac{\mathrm{i}}{2} \mathrm{~d} K=\frac{1}{2}\left(P_{1} \mathrm{~d} Q_{1}+P_{2} \mathrm{~d} Q_{2}-Q_{1} \mathrm{~d} P_{1}-Q_{2} \mathrm{~d} P_{2}\right)
$$


In the gauge given by $\theta$ the wave functions can be expressed by

$$
\psi(z, \bar{z})=\phi(z) \mathrm{e}^{-|z|^{2} / 4 \hbar}
$$

where $\phi$ is a holomorphic function of $z_{1}$ and $z_{2}$. The Hilbert space of square integrable wave functions have scalar product

$$
\left\langle\psi_{1}, \psi_{2}\right\rangle_{K}=\frac{1}{(2 \pi \hbar)^{2}} \int_{\mathbb{C}^{2}} \overline{\phi_{1}(z)} \phi_{2}(z) \mathrm{e}^{-|z|^{2} / 2 \hbar} \mathrm{d}^{2} z
$$

where $\mathrm{d}^{2} z$ is the Lebesgue measure in $\mathbb{C}^{2}$, which coincides with the Liouville volume.

The holomorphic function $\phi$ is an element of what is known as the Segal-Bargmann space or Bargmann-Fock space [3,12,24]. This space is a closed subspace of the Hilbert space of square integrable functions on $\mathbb{C}^{2}$ with the Gaussian measure. Therefore, it is a Hilbert space denoted $\mathcal{H}_{K}$.

One of the most important characteristics of these spaces is that the point evaluation operator is continuous. Hence, there exists a function named reproducing kernel, holomorphic in the first variable and antiholomorphic in the second one, which in this case is $\mathrm{e}^{w \cdot \bar{v} / 2 \hbar}$ and for all $\phi \in \mathcal{H}_{K}$ verifies

$$
\phi(w)=\frac{1}{(2 \pi \hbar)^{2}} \int_{\mathbb{C}^{2}} \phi(v) \mathrm{e}^{w \cdot \bar{v} / 2 \hbar} \mathrm{e}^{-|v|^{2} / 2 \hbar} \mathrm{d}^{2} v
$$

where $w \cdot \bar{v}=w_{1} \bar{v}_{1}+w_{2} \bar{v}_{2}$.

To find the operators corresponding to $H$ and $L$ we must find the corresponding Hamiltonian fields. From $\omega\left(X_{f}, \cdot\right)=\mathrm{d} H$ and $\omega\left(X_{f}, \cdot\right)=\mathrm{d} L$ we obtain

$$
\begin{aligned}
& X_{H}=\mathrm{i} \omega_{c}\left(z_{1} \frac{\partial}{\partial z_{1}}-\bar{z}_{1} \frac{\partial}{\partial \bar{z}_{1}}\right) \\
& X_{L}=\mathrm{i}\left(z_{2} \frac{\partial}{\partial z_{2}}-z_{1} \frac{\partial}{\partial z_{1}}\right)-\mathrm{i}\left(\bar{z}_{2} \frac{\partial}{\partial \bar{z}_{2}}-\bar{z}_{1} \frac{\partial}{\partial \bar{z}_{1}}\right) .
\end{aligned}
$$

The terms in the conjugate directions vanish in the space of polarized functions. Then, the quantized operators $\hat{H}$ and $\hat{L}$, obtained using geometric quantization with half-form correction (8) in the gauge given by $\theta$ are given by

$$
\begin{aligned}
& \hat{H} \psi=\hbar \omega_{c}\left(z_{1} \frac{\partial \phi}{\partial z_{1}}+\frac{1}{2} \phi\right) \mathrm{e}^{-|z|^{2} / 4 \hbar} \\
& \hat{L} \psi=\hbar\left(z_{2} \frac{\partial \phi}{\partial z_{2}}-z_{1} \frac{\partial \phi}{\partial z_{1}}\right) \mathrm{e}^{-|z|^{2} / 4 \hbar} .
\end{aligned}
$$

The $1 / 2$ term in $\hat{H}$ arises from the Lie derivative of the square root of the volume as explained above. 


\subsection{The Spectrum of $\hat{H}$ and $\hat{L}$}

The operator $\hat{H}$ is the Hamiltonian of a harmonic oscillator and its holomorphic eigenfunctions are positive or zero powers of the variable $z_{1}$. In particular, the equation $\hat{H} \psi=E \psi$ leads to the differential equation

$$
z_{1} \frac{\partial \phi}{\partial z_{1}}+\frac{1}{2} \phi=\frac{E}{\hbar \omega_{c}} \phi
$$

whose holomorphic solutions are of the form $\phi_{n}=z_{1}^{n} g\left(z_{2}\right)$. Then, we have

$$
\begin{aligned}
\hat{H} \psi & =\hbar \omega_{c}\left(n+\frac{1}{2}\right) \psi \\
\hat{L} \psi & =\hbar z_{1}^{n}\left(z_{2} \frac{\mathrm{d} g}{\mathrm{~d} z_{2}}-n g\right) \mathrm{e}^{-|z|^{2} / 4 \hbar} .
\end{aligned}
$$

Let $l$ be the eigenvalue of the angular momentum, i.e., $\hat{L} \psi=l \psi$. Then, we have

$$
z_{2} \frac{\mathrm{d} g}{\mathrm{~d} z_{2}}-n g=l g
$$

Solving this equation for holomorphic $g$ we obtain the normalized eigenfunctions

$$
\psi_{n l}(z)=\frac{z_{1}^{n} z_{2}^{n+l}}{(2 \hbar)^{n+l / 2} \sqrt{n !(n+l) !}} \mathrm{e}^{-|z|^{2} / 4 \hbar}
$$

where $l$ takes all integer values from $-n$. This is the Landau spectrum (see for example Landau and Lifshitz [19]).

\section{The Segal-Bargmann Transform}

We were interested in finding the unitary map between the Schrödinger representation in the original canonical coordinates $(q, p)$ and the holomorphic representation. First we made a unitary transformation on $\mathbb{C}^{2}$ to transform the coordinates $\left(z_{1}, z_{2}\right)$ into new coordinates $\left(w_{1}, w_{2}\right)$ preserving the polarization. The change is given by

$$
w_{1}=\frac{z_{1}+\mathrm{i} z_{2}}{\sqrt{2}}, \quad w_{2}=\frac{z_{2}+\mathrm{i} z_{1}}{\sqrt{2}} .
$$

Then, the wave functions are given by

$$
\psi(w)=\phi(w) \mathrm{e}^{-|w|^{2} / 4 \hbar}
$$

with the scalar product (22). 
The new coordinates $w_{1}$ and $w_{2}$ allowed us to relate more easily the holomorphic representation with the original coordinates $(q, p)$ (9). The real part of $w$ is $p$ and the imaginary part is $q$, both up to a constant.

The common eigenvectors of $\hat{H}$ and $\hat{L}$ are

$$
\psi_{n l}(w)=\frac{\left(w_{1}-\mathrm{i} w_{2}\right)^{n}\left(w_{2}-\mathrm{i} w_{1}\right)^{n+l}}{(4 \hbar)^{n+l / 2} \sqrt{n !(n+l) !}} \mathrm{e}^{-|w|^{2} / 4 \hbar} .
$$

Following, we studied the pairing between this Hilbert space and the space corresponding to the real polarization used in the Schrödinger representation. In this polarization the leaves are characterized by constant $q$ coordinates, so the polarized functions are only functions of $q_{1}$ and $q_{2}$.

The one-form $\theta$ that we used to represent the polarized wave functions in the holomorphic representation is invariant under the coordinate transformation which sends the original $(q, p)$ to $(Q, P)$. Thus we have

$$
\begin{aligned}
\theta & =\frac{1}{2}\left(P_{1} \mathrm{~d} Q_{1}+P_{2} \mathrm{~d} Q_{2}-Q_{1} \mathrm{~d} P_{1}-Q_{2} \mathrm{~d} P_{2}\right) \\
& =\frac{1}{2}\left(p_{1} \mathrm{~d} q_{1}+p_{2} \mathrm{~d} q_{2}-q_{1} \mathrm{~d} p_{1}-q_{2} \mathrm{~d} p_{2}\right) \\
& =\theta_{0}+\mathrm{d} u=\theta_{K}+\frac{\mathrm{i}}{2} \mathrm{~d} K .
\end{aligned}
$$

where $\theta_{0}=p_{1} \mathrm{~d} q_{1}+p_{2} \mathrm{~d} q_{2}$ is the canonical one-form and $u=-\left(q_{1} p_{1}+q_{2} p_{2}\right) / 2$. So the polarized wave functions in the Schrödinger representation and the holomorphic representation are

$$
\psi_{S}(q, p)=\phi_{S}(q) \mathrm{e}^{-\mathrm{i} q \cdot p / 2 \hbar}, \quad \psi_{K}(w, \bar{w})=\phi_{K}(w) \mathrm{e}^{-|w|^{2} / 4 \hbar} .
$$

One remarkable observation in the last line of the first equation in (33) is that the selection of the coordinate $z=P+\mathrm{i} Q$ instead of $Q+\mathrm{i} P$ is responsible for the compatibility of the forms $\theta_{K}$ and $\theta_{0}$. Therefore, a pairing between representations can be readily written. This selection is somewhat arbitrary; however, this change leads to a similar formula albeit with a change of the sign for the term $\theta_{0}$. Then, we must also change the sign of the symplectic form.

As stated earlier, the complex variable $w$ can be expressed as follows in terms of the original canonical variables

$$
\begin{aligned}
& w_{1}=\frac{\sqrt{2}}{\sqrt{e B}} p_{1}+\mathrm{i} \frac{\sqrt{e B}}{\sqrt{2}} q_{1} \\
& w_{2}=\frac{\sqrt{2}}{\sqrt{e B}} p_{2}+\mathrm{i} \frac{\sqrt{e B}}{\sqrt{2}} q_{2} .
\end{aligned}
$$


The product of $\psi_{S}$ with $\psi_{K}$ can be integrated on the original manifold with the Liouville volume. Then, we defined a pairing between these representations as follows

$$
\left\langle\psi_{S}, \psi_{K}\right\rangle_{S K}=\frac{1}{(2 \pi \hbar)^{2}} \int_{\mathbb{C}^{2}} \overline{\phi_{S}(q)} \phi_{K}(w) \mathrm{e}^{\mathrm{i} q \cdot p / 2 \hbar-|w|^{2} / 4 \hbar} \mathrm{d}^{2} p \mathrm{~d}^{2} q .
$$

Using the property (23) for $\phi_{K}$ and integrating in the variables $p_{1}$ and $p_{2}$ we have

$$
\left\langle\psi_{S}, \psi_{K}\right\rangle_{S K}=\frac{1}{(2 \pi \hbar)^{2}} \int_{\mathbb{C}^{2} \times \mathbb{R}^{2}} \overline{\phi_{S}(q)} \phi_{K}(v) \overline{G(q, v)} \mathrm{e}^{-|v|^{2} / 2 \hbar} \mathrm{d}^{2} q \mathrm{~d}^{2} x \mathrm{~d}^{2} y .
$$

where $G$ is the function

$$
G(q, v)=\frac{\mathrm{e}^{-e B(q-\mathrm{i} \sqrt{2} v)^{2} / 8 \hbar} \mathrm{e}^{-e B q \cdot(q-\mathrm{i} 2 \sqrt{2} v) / 8 \hbar}}{2 \pi \hbar} .
$$

For convenience we used the integration variables

$$
v_{1}=\sqrt{\frac{e B}{2}}\left(2 x_{1}+\mathrm{i} y_{1}\right) \quad \text { and } \quad v_{2}=\sqrt{\frac{e B}{2}}\left(2 x_{2}+\mathrm{i} y_{2}\right)
$$

This pairing naturally induces a unitary map between holomorphic representation and Schrödinger representation by imposing compatibility with scalar products (22) of $\mathcal{H}_{K}$ and

$$
\left\langle\psi_{1}, \psi_{2}\right\rangle_{S}=\frac{e B}{2 \pi \hbar} \int_{\mathbb{R}^{2}} \overline{\psi_{1}(q)} \psi_{2}(q) \mathrm{d}^{2} q
$$

of $\mathcal{H}_{S}$ (the latter is, up to a constant, the usual product in $L^{2}\left(\mathbb{R}^{2}\right) \equiv L^{2}(\mathbb{C})$ ). Then, it must be verified that

$$
\left\langle\psi_{S}, \psi_{K}\right\rangle_{S K}=\left\langle\psi_{S}, \Lambda \psi_{K}\right\rangle_{S}=\left\langle\Lambda^{-1} \psi_{S}, \psi_{K}\right\rangle_{K}
$$

These identities hold for isometries

$$
\left(\Lambda \psi_{K}\right)(q)=\frac{1}{2 \pi \hbar} \int_{\mathbb{C}^{2}} \phi_{K}(v) \overline{G(q, v)} \mathrm{e}^{-|v|^{2} / 2 \hbar} \mathrm{d}^{2} x \mathrm{~d}^{2} y
$$

and $\Lambda^{-1}$, which is given by

$$
\left(\Lambda^{-1} \psi_{S}\right)(w)=\frac{1}{2 \pi \hbar} \int_{\mathbb{R}^{2}} \phi_{S}(q) G(q, w) \mathrm{d}^{2} q
$$

The above expressions are versions of the Segal-Bargmann transform [3, 12, 24, 29, 31]. 
Using the equation (42) we can find the wave functions in the Schrödinger representation that corresponds to the eigenfunctions $\psi_{n l}$ shown in (32). In terms of the complex variable $\xi=\sqrt{e B}\left(q_{1}+\mathrm{i} q_{2}\right) / \sqrt{2 \hbar}$ we obtain

$$
\left(\Lambda \psi_{n l}\right)(q)=(-\mathrm{i})^{n} \frac{\sqrt{n !}}{\sqrt{(n+l) !}} \xi^{l} L_{n}^{l}\left(|\xi|^{2}\right) \mathrm{e}^{-e B\left(q_{1}^{2}+q_{2}^{2}\right) / 2 \hbar}
$$

where $L_{n}^{l}$ is a generalized Laguerre polynomial. These eigenfunctions coincide with Dunne's [6] for the same problem.

\section{Conclusions}

In the literature we can find interesting examples of complex problems solved through a specific coordinate transformations, e.g. [7]. Guided by the rules of geometric quantization, we show how to analyze in detail the structure of the Hilbert space of a magnetic problem by using a formalism of holomorphic functions, with an original approach involving a specific change of coordinates. Moreover, our work allows for the relation of different representations associated with the problem.

\section{Acknowledgements}

The work is supported by the Universidad Nacional del Sur (Grant \# PGI 24/L096).

\section{References}

[1] Abraham R. and Marsden J., Foundations of Mechanics, $2^{\text {nd }}$ Edn, AddisonWesley, Redwood 1987.

[2] Arnold V., Mathematical Methods of Classical Mechanics, Graduate Texts in Mathematics 60, Springer, New York 1978.

[3] Bargmann V., On a Hilbert Space of Analytic Functions and an Associated Integral Transform, Comm. Pure Appl. Math. 14 (1961) 187-214.

[4] de Brito P. and Nazareno H., Particle in a Uniform Magnetic Field Under the Symmetric Gauge: The Eigenfunctions and the Time Evolution of Wave Packets, Europ. J. Phys. 28 (2007) 9-21.

[5] Cushman R. and Śniatycki J., On Bohr-Sommerfeld-Heisenberg Quantization J. Geom. Symmetry Phys. 35 (2014) 11-19. 
[6] Dunne G., Hilbert Space for Charged Particles in Perpendicular Magnetic Fields, Ann. Phys. 215 (1992) 233-263.

[7] Duru I. and Kleinert H., Solution of the Path Integral for the H-Atom, Physics Letters B 84 (1979) 185-188.

[8] Gotay M. and Isenberg J., The Symplectization of Science, Symplectic Geometry Lies at the Very Foundations of Physics and Mathematics, Gazette des Mathématiciens 54 (1992) 59-79.

[9] Griffiths P. and Harris J., Principles of Algebraic Geometry, John Wiley \& Sons, New York 1978.

[10] Groenewald H., On the Principles of Elementary Quantum Mechanics, Physica 12 (1946) 405-460.

[11] Guillemin V. and Sternberg S., Geometric Asymptotics, Mathematical Surveys 14, AMS, Providence 1977.

[12] Hall B., Holomorphic Methods in Mathematical Physics, In: First Summer School in Analysis and Mathematical Physics (Cuernavaca Morelos, 1998), S. Pérez-Esteva and C. Vilegas-Blas (Eds), Amer. Math. Soc., Providence 2000, pp 1-59.

[13] Hall B., Quantum Theory for Mathematicians, Springer, New York 2013.

[14] Hall B. and Kirwin W., Adapted Complex Structures and the Geodesic Flow, Math. Ann. 350 (2011) 455-474.

[15] Hurt N., Geometric Quantization in Action, Reidel, Dordrecht 1982.

[16] Kirillov A., Geometric Quantization, Springer, Berlin 1990.

[17] Kobayashi S. and Nomizu K., Foundations of Differential Geometry vol. 2, Interscience Publishers, John Wiley \& Sons, New York 1969.

[18] Kostant B., Quantization and Unitary Representations, LNM 170, Springer, New York 1970.

[19] Landau L. and Lifshitz E., Quantum Mechanics. Non-Relativistic Theory, $2^{\text {nd }}$ Edn, Course of Theoretical Physics 3, Pergamon Press, Oxford 1965.

[20] Laughlin R., Quantized Hall Conductivity in Two Dimensions, Phys. Rev. B 23 (1981) 5632-5633.

[21] Newlander A. and Niremberg L., Complex Coordinates in Almost Complex Manifolds, Ann. Math. 65 (1957) 391-404.

[22] Puta M., Hamiltonian Mechanical Systems and Geometric Quantization, Kluwer, Dordrecht 1993.

[23] Rosenberg J., A Selective History of the Stone-von Neumann Theorem, Contemp. Math. 365 (2004) 331-354. 
[24] Segal I. and Mackey G., Mathematical Problems of Relativistic Physics, Lectures in Applied Mathematics II, American Mathematical Society, Providence 1963.

[25] Simms D. and Woodhouse N., Lectures on Geometric Quantization, Lecture Notes in Physics 53, Springer, Berlin 1976.

[26] Śniatycki J., Bohr-Sommerfeld Conditions in Geometric Quantization, Rep. Math. Phys. 7 (1975) 303-311.

[27] Śniatycki J., Geometric Quantization and Quantum Mechanics, Springer, Berlin 1980.

[28] Souriau J.-M., Structure des Systèmes Dynamiques, Dunod, Paris 1970.

[29] Stenzel M., The Segal-Bargmann Transform on a Symmetric Space of Compact Type, J. Funct. Anal. 165 (1999) 44-58.

[30] Wilczek F., Quantum mechanics of Fractional-Spin Particles, Phys. Rev. Lett. 49 (1982) 957-959.

[31] Woodhouse N., Geometric Quantization, $2^{\text {nd }}$ Edn, Clarendon Press, Oxford 1991.

\section{Received 26 January 2016}

Guillermo Capobianco

Departamento de Matemática

Universidad Nacional del Sur

Av. Alem 1253, 8000 Bahía Blanca

Buenos Aires, ARGENTINA

E-mail address: guillermo.capobianco@gmail.com

Walter Reartes

Departamento de Matemática

Universidad Nacional del Sur

Av. Alem 1253, 8000 Bahía Blanca

Buenos Aires, ARGENTINA

E-mail address: walter.reartes@gmail.com 